\title{
Right and left-sided carcinoid heart disease
}

\author{
Ilona M. Michałowska, Agnieszka Kolasińska-Ćwikla, Jarosław Ćwikla, \\ Dariusz Zakrzewski, Mariola Pęczkowska
}

Institute of Cardiology, Warsaw, Poland

A 50-year-old woman with carcinoid - midgut neuroendocrine neoplasm (NET G1, Ki-67 = 1\%), and metastases to regional lymph nodes was admitted to the hospital due to deterioration in exercise tolerance and leg swelling. She suffered from sclerosis multiplex, glaucoma and depression. She had been treated for depressive episodes with selective serotonin re-uptake inhibitors (SSRIs) before the development of carcinoid.

Transthoracic echocardiography revealed enlargement of the atria and right ventricle (RV), severe mitral and tricuspid valve (MV, TV) regurgitation with leaflet thickening (Fig. 1A-C).

A mean mitral gradient was $8 \mathrm{mmHg}$, the mitral valve area $1.5 \mathrm{~cm}^{2}$ with normal ejection fraction of the left ventricle. Right and left-sided carcinoid heart disease was recognized. Cardiac computed tomography (CCT) was performed in order to evaluate coronary arteries and carcinoid heart involvement before surgery.
The coronary arteries were normal. The CCT showed biatrial enlargement, thickening of MV and TV including leaflets, chordae, and papillary muscles with incomplete coaptation and a mild thickening of the pulmonary valve (Fig. 1D-F). Patent foramen ovale was not detected.

Carcinoid heart disease occurs in patients with liver or retroperitoneal lymph node metastases as well as in those with primary ovarian or pulmonary carcinoid.

This is the unique case of a patient with carcinoid located in the Bauchin valve and metastases only to regional lymph nodes who was treated with SSRIs and developed bilateral carcinoid heart disease. Several case reports suggest that SSRIs may function as a provocative agent of carcinoid syndrome and/or deterioration of carcinoid syndrome and carcinoid heart disease.

\section{Conflict of interest: None declared}

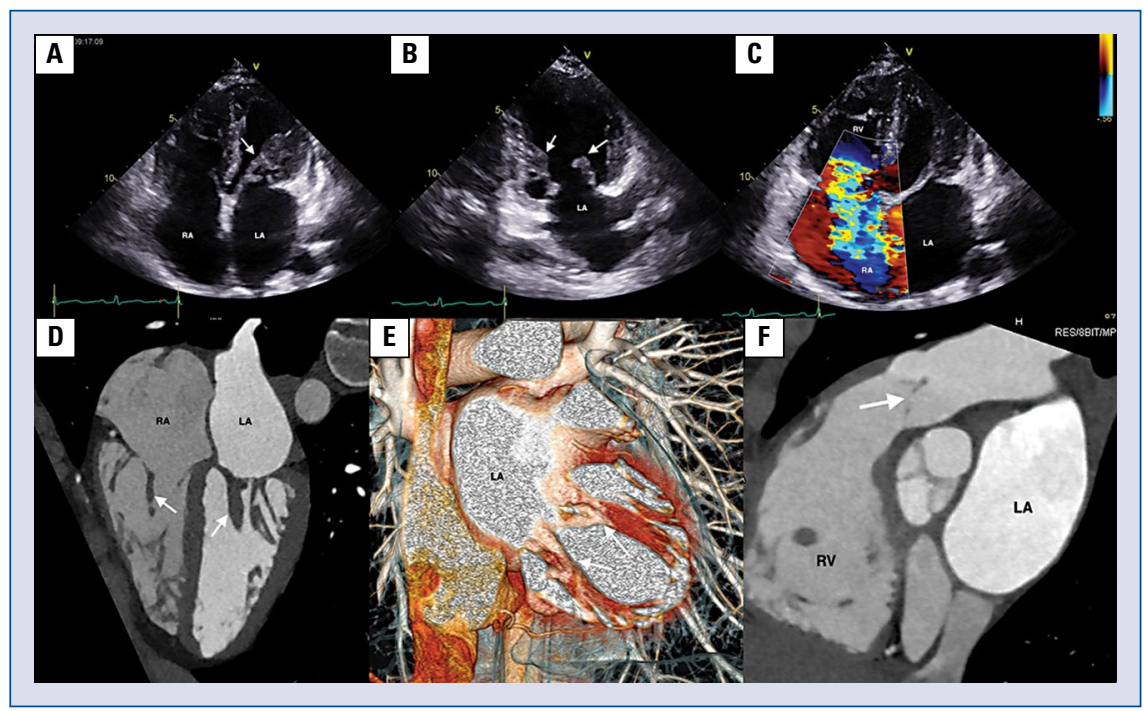

Figure 1. A-C. Transthoracic echocardiography shows thickening of the mitral valve leaflets and apparatus (arrows) as well as severe tricuspid regurgitation; D-F. Cardiac computed tomography shows thickening of mitral and tricuspid valve apparatus and thickening of the pulmonary valve leaflets (arrows); LA — left atrium; RA — right atrium.

Address for correspondence: Ilona M. Michałowska, PhD, MD, Institute of Cardiology, ul. Alpejska 42, 04-628 Warszawa, Poland, tel: +48 2234341 68, e-mail: ilonamich@wp.pl

Received: 20.12.2017 Accepted: 16.01.2018 